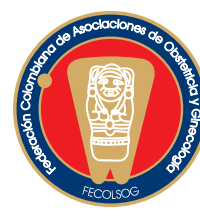

\title{
LAS INFECCIONES DEL TRACTO GENITAL FEMENINO: UNA OPORTUNIDAD PARA RENOVAR LAS POLÍTICAS Y LA INVESTIGACIÓN EN SALUD SEXUAL Y REPRODUCTIVA
}

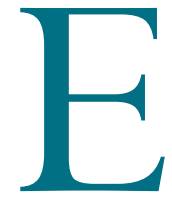

n este número de la Revista Colombiana de Obstetricia y Ginecología se presentan los resultados del estudio: "Prevalencia y factores asociados a la infección por $C$. trachomatis, N. gonorrheae, $T$. vaginalis, C. albicans, sífilis, VIH y vaginosis bacteriana en mujeres con síntomas de infección vaginal en tres sitios de atención de Bogotá, Colombia, 2010”. Estos resultados muestran a la vaginosis bacteriana y Candida sp. como las principales causas de estas infecciones. Respecto a las ITS se encontró que Chlamydia trachomatis fue el germen con mayor prevalencia. Además, se determinó que Neisseria gonorroeae, Treponema pallidum y Trichomonas vaginalis siguen siendo frecuentes en nuestra población, no obstante su baja prevalencia.

Este estudio nos alerta sobre la importancia que siguen teniendo las infecciones del tracto genital como problema de salud pública en el país, no solo por el consumo de recursos relacionados con la consulta al médico general o especialista, los métodos diagnósticos utilizados y el tratamiento, entre otros, sino también por las consecuencias en calidad de vida, las complicaciones y las secuelas en las pacientes. Por otra parte, en nuestro medio es posible que se presente amplia variabilidad en la práctica clínica respecto al diagnóstico y manejo de estas infecciones.

Con base en estos hechos, el Ministerio de Salud hizo una convocatoria para el desarrollo de la Guía de Atención Integral para el manejo sindrómico de las ITS (1) con el ánimo de actualizar la guía que fue publicada en la resolución 412 del año 2000, que daba las pautas para el manejo de las ITS en el país (2).
Los resultados de este estudio brindan información para orientar y definir políticas de investigación y atención en salud pública alrededor del tema de las ITS no VIH-SIDA, y servirá de apoyo para definir qué entidades es conveniente abordar con enfoque sindrómico y cuáles con abordaje etiológico fundamentado en pruebas de laboratorio, ya sean pruebas rápidas para ser realizadas en el sitio de atención del paciente, o pruebas convencionales que requieren una cita posterior para su interpretación. Este estudio también abre la posibilidad de vislumbrar la conveniencia de realizar investigaciones de base poblacional que incluyan varias zonas del país, donde se estudie la magnitud del problema y sus consecuencias clínicas, sociales y económicas en poblaciones especiales como las gestantes, los y las adolescentes, y la mujer asintomática. En este último grupo son de especial importancia las ITS por gonococo y clamidia ya que en él cursan frecuentemente de manera asintomática, pero con gran potencial de daño sobre la función reproductiva de la mujer.

De manera simultánea hemos recibido la buena noticia de que la Colaboración Cochrane ha seleccionado a Colombia, a través del Instituto de Investigaciones Clínicas de la Universidad Nacional, para coordinar el grupo revisor de Infecciones de Transmisión Sexual. La Colaboración Cochrane es una entidad internacional sin ánimo de lucro, creada en 1993, cuyo objetivo es ayudar a los proveedores de servicios de salud, a quienes definen las políticas en salud, a los pacientes y sus cuidadores en la toma de decisiones relacionadas con los problemas de salud que se enfrentan, ya sea a nivel individual o comunitario, con base en la mejor 
evidencia disponible por medio de la preparación, actualización y promoción del acceso a las Revisiones Cochrane (3). Estas revisiones son referente internacional de alta calidad en la información acerca de la efectividad en el cuidado de la salud.

El reconocimiento del problema que siguen representando las infecciones del tracto genital en nuestras pacientes, la próxima publicación de la guía de atención integral de estas infecciones, y el registro del grupo revisor en ITS de la Colaboración Cochrane, dan la oportunidad de posicionar nuevamente el tema de las infecciones del tracto genital en la agenda nacional de salud pública.

Se solicita entonces que el Gobierno nacional provea la normatividad y vigilancia que obligue a las entidades promotoras de salud (EPS) de los regímenes contributivos y subsidiados, y a los gobiernos locales, a destinar los recursos necesarios para cubrir de manera adecuada los servicios de salud sexual y reproductiva que involucren la prevención de estas infecciones, así como el cuidado de estas pacientes y sus contactos. Esto es vital para cortar la cadena epidemiológica de transmisión. Por otra parte, se requiere una adecuada oferta de servicios que permitan la prevención de esas infecciones mediante actividades educativas que reduzcan el riesgo de adquirirlas, en grupos especialmente vulnerables como los adolescentes o las comunidades LGBT, o al momento del contacto de los pacientes con los servicios de salud como son el control prenatal o la consulta de planificación. Se requiere que las instituciones tengan la capacidad resolutiva para un diagnóstico temprano y un tratamiento oportuno que garantice la adherencia, a fin de evitar las recurrencias y la aparición de resistencia a los antibióticos, y que además provean la asesoría psicológica de apoyo y el seguimiento adecuado de los pacientes que así lo requieran.

Por otra parte, se requiere que el Instituto Nacional de Salud reinicie las actividades de vigilancia y control de las ITS - más allá de la vigilancia del VIHSIDA-, inicialmente a través de actividades de reporte de los diferentes síndromes y, a mediano plazo, me- diante la instauración de tecnologías que nos permitan hacer el correcto diagnóstico etiológico. También es necesario tener una red de laboratorios de salud pública que permitan conocer las tasas de resistencia de los diversos microorganismos a los antibióticos de elección en las diferentes regiones del país.

Nos corresponde, como obstetras y ginecólogos, o como médicos generales, capacitarnos adecuadamente en las actividades de promoción, prevención y atención de estos pacientes con infecciones del tracto genital, como también llevar a cabo investigaciones que permitan conocer la magnitud del problema, plantear intervenciones efectivas y seguras que reduzcan su frecuencia, y minimizar las consecuencias en nuestra comunidad. Como Federación nos corresponde hacer el control social para que los diferentes actores del sistema cumplan con su responsabilidad, y liderar acciones que vayan en bien de la salud de nuestras mujeres y sus hijos, actores secundarios en esta problemática.

\section{Hernando Gaitán MD MSc}

Editor

\section{Santiago Estrada MD}

Editor invitado

Director general

Laboratorio Clínico Congregación Mariana

\section{REFERENCIAS}

1. Colciencias. Convocatoria 500 de 2009. Convocatoria para la conformación de un banco de proyectos para el desarrollo de Guías de Atención Integral (GAI) basadas en la evidencia. Disponible en: http://www.colciencias. gov.co/convocatoria/convocatoria-para-la-conformacin-de-un-banco-de-proyectos-para-el-desarrollo-degu-de-

2. República de Colombia. Ministerio de Salud. Dirección General de Promoción y Prevención. Normas técnicas y guías de atención. Resolución 412/2000.

3. Cochrane Collaboration. Tomado el 16 de abril de 2012. Disponible en: http://www.cochrane.org/ 


\section{NOTA DEL EDITOR}

En este número de la Revista Colombiana de Obstetricia y Ginecología se publica por primera vez un artículo de investigación original en español e inglés. Se busca bajo esta estrategia tener una mayor difusión de nuestra investigación a nivel internacional, dado que el inglés es el idioma universal de la ciencia. La Revista Colombiana de Anestesia es pionera en este esfuerzo, financia la traducción y publica en la actualidad todos los artículos en los dos idiomas. Es nuestra decisión ir en esta dirección, y aunque nuestras limitaciones financieras no nos permiten cubrir el costo de la traducción, sí publicaremos a partir de la fecha los manuscritos que sean enviados por los autores en los dos idiomas. Invitamos a nuestros autores a apoyarnos en este esfuerzo para lograr que nuestras investigaciones no solo sean leídas por nuestro público en Latinoamérica, sino también en países no hispano-parlantes, y así tengan una mayor cobertura.

\section{Hernando Gaitán MD MSc}

Editor 